\title{
BMJ Open Rationalisations for women-only randomised controlled trials in conditions that affect both sexes: a scoping review protocol
}

\author{
Ainsley Matthewson (D) ,, ${ }^{1,2}$ Olena Bereznyakova, ${ }^{3}$ Brian Dewar, ${ }^{2}$ Alexandra Davis, ${ }^{2}$ \\ Mark Fedyk, ${ }^{4}$ Vignan Yogendrakumar (D) ,1,2 Dean A Fergusson (D) , \\ Sophia Gocan, ${ }^{2}$ Dar Dowlatshahi, ${ }^{1,2}$ Robert Fahed, ${ }^{1,2}$ Michel Shamy ${ }^{1,2}$
}

To cite: Matthewson A, Bereznyakova 0, Dewar B, et al. Rationalisations for women-only randomised controlled trials in conditions that affect both sexes: a scoping review protocol. BMJ Open 2021;11:e043370. doi:10.1136/ bmjopen-2020-043370

- Prepublication history and additional material for this paper is available online. To view these files, please visit the journal online (http://dx.doi.org/10. 1136/bmjopen-2020-043370).

Received 10 August 2020 Revised 16 January 2021 Accepted 22 January 2021

\section{Check for updates}

(C) Author(s) (or their employer(s)) 2021. Re-use permitted under CC BY-NC. No commercial re-use. See rights and permissions. Published by BMJ.

${ }^{1}$ Faculty of Medicine, University of Ottawa, Ottawa, Ontario, Canada

${ }^{2}$ Ottawa Hospital \& Ottawa Hospital Research Institute, Ottawa, Ontario, Canada

${ }^{3}$ Department of Neurosciences, Centre Hospitalier de I'Université de Montréal, Montreal, Québec, Canada

${ }^{4}$ Betty Irene Moore School of Nursing, University of California, Davis, California, USA

Correspondence to Ms Ainsley Matthewson; amatt074@uottawa.ca

\section{ABSTRACT}

Introduction Women have historically been underrepresented in randomised controlled trials (RCTs), including many landmark RCTs that established standards of care. In light of this fact, some modern researchers are calling for replication of earlier landmark trials with women only. This approach is ethically concerning, in that it would require some enrolled women to be deprived of treatments that are currently considered standard of care.

Objective In an attempt to better understand the justification of a women-only approach to designing clinical trials, this study looks to systematically categorise the number of women-only RCTs for conditions that affect both men and women and the reasons given within the medical and philosophical literatures to perform them. Methodology This scoping review of the literature will search, screen and select articles based on predetermined inclusion/exclusion criteria, after which a grounded theory approach will be used to synthesise the data. It is expected that there will be a variety of reasons given for why a women-only trial may be justified. Electronic databases that will be searched include MEDLINE, EMBASE, Cochrane Database of Systematic Reviews, Cochrane Clinical Trials Register, Web of Science Proceedings, ClinicalTrials. gov, Philosopher's Index, Phil Papers, JSTOR, Periodicals Archive Online, Project MUSE and the National Reference Centre for Bioethics.

Significance The scope of this study is to determine published rationales used to justify women-only randomised trials, both in the case of new trials and in the repetition of landmark trials.

Ethics and dissemination Research ethics board approval is not required for this study as there is no participant involvement. Results will be published as a stand-alone manuscript and will inform a larger project related to the ethics of a women-only RCT of carotid intervention for women with symptomatic high-grade carotid stenosis.

\section{INTRODUCTION}

Randomised controlled trials (RCTs) are widely viewed as the best way to test treatments in medicine; ${ }^{1}$ however, women have been historically underrepresented in RCTs,
Strengths and limitations of this study

- Will help bridge a gap in medical knowledge regarding the ethical and scientific justification of womenonly randomised controlled trials (RCTs).

- Will contribute to developing a framework for considering the ethical and scientific justification of women-only RCTs in conditions that affect both women and men.

- Will inform a larger project related to the ethics of a women-only RCT of carotid intervention for women with symptomatic high-grade carotid stenosis.

- The process of categorising the reasons given to justify women-only RCTs neither ensure that they are good reasons nor that they are the only possible reasons for women-only RCTs.

- Employing the framework method is a complex approach that is resource intensive and timeconsuming and requires experienced researchers for its successful implementation.

including in many landmark RCTs that are known to have established currently accepted standards of care. For example, underrepresentation of women in RCTs is seen widely across cardiovascular disease prevention trials that inform women-specific guidelines, with women making up only $30 \%$ of enrolled patients. Notably, female enrolment in stroke trials is only $38 \%{ }^{2}$

In light of this fact, researchers in some disciplines including stroke neurology are calling for the replication of earlier landmark trials with women only. ${ }^{3}$ This issue has recently arisen in the literature surrounding the management of women with carotid stenosis, a condition that puts patients with recent transient ischaemic attack or minor stroke at high risk for recurrent, major stroke. ${ }^{4}$ We are unsure if this same issue has arisen in other disciplines, and if so, how it has been addressed. Calls for women-only 
trials seem to be rare in contrast to calls that focus on the inclusion of more women in RCTs and sex-specific analysis of the data. ${ }^{56}$

The concept of performing a women-only RCT in a condition for which a standard of care with a strong evidence base may already exist presents an ethical challenge, in that it would require some enrolled women to be deprived of that standard of care for the purposes of completing a trial.

In order to avoid unjustifiably performing a womenonly RCT, researchers, regulators and funders should be able to refer to a conceptual framework if not specific criteria to ensure that women-only RCTs are scientifically and ethically justified. It is currently unclear what those criteria might be. This work will characterise how many women-only RCTs have been conducted in conditions affecting both men and women, will seek or demonstrate how often reasons have been used to justify these womenonly trials and what these reasons are. Example conditions include diseases of the nervous and cardiovascular systems as well as certain cancers. Urogenital conditions, cosmetic procedures and other conditions with a marked sex difference are not relevant to our research question.

\section{OBJECTIVES}

Due to the variety and complexity of sources, a scoping review was chosen over a systematic review to be fully inclusive in the review, that is, cover the whole body of literature. We aim to conduct a scoping review of the medical and philosophical literatures to answer the following questions:

1. How many women-only trials have been conducted in conditions affecting both women and men?

2. How often are explicit reasons offered as justification for a women-only trial in a condition affecting women and men?

3. What are the reasons offered?

A scoping review will map the literature to determine what, if anything, is known about our research questions. A systematic review and quantitative meta-analysis are not required as they will not inform our specific questions. We hypothesise that there will be a relatively small number of women-only RCTs in conditions affecting both women and men, with few offering explicit reasons for the trials. Among those reasons, we expect that there will be significant heterogeneity across the literature.

\section{METHODS AND ANALYSIS}

This study follows the reported guidance for conducting systematic scoping reviews. ${ }^{7}$ The study will be performed in three stages: article search and selection, data extraction and analysis (figure 1).

The article search process will combine search terms meaning 'women' and its variants with search terms describing 'randomised clinical trials' and its variants. From this set of results, studies that contain variations of

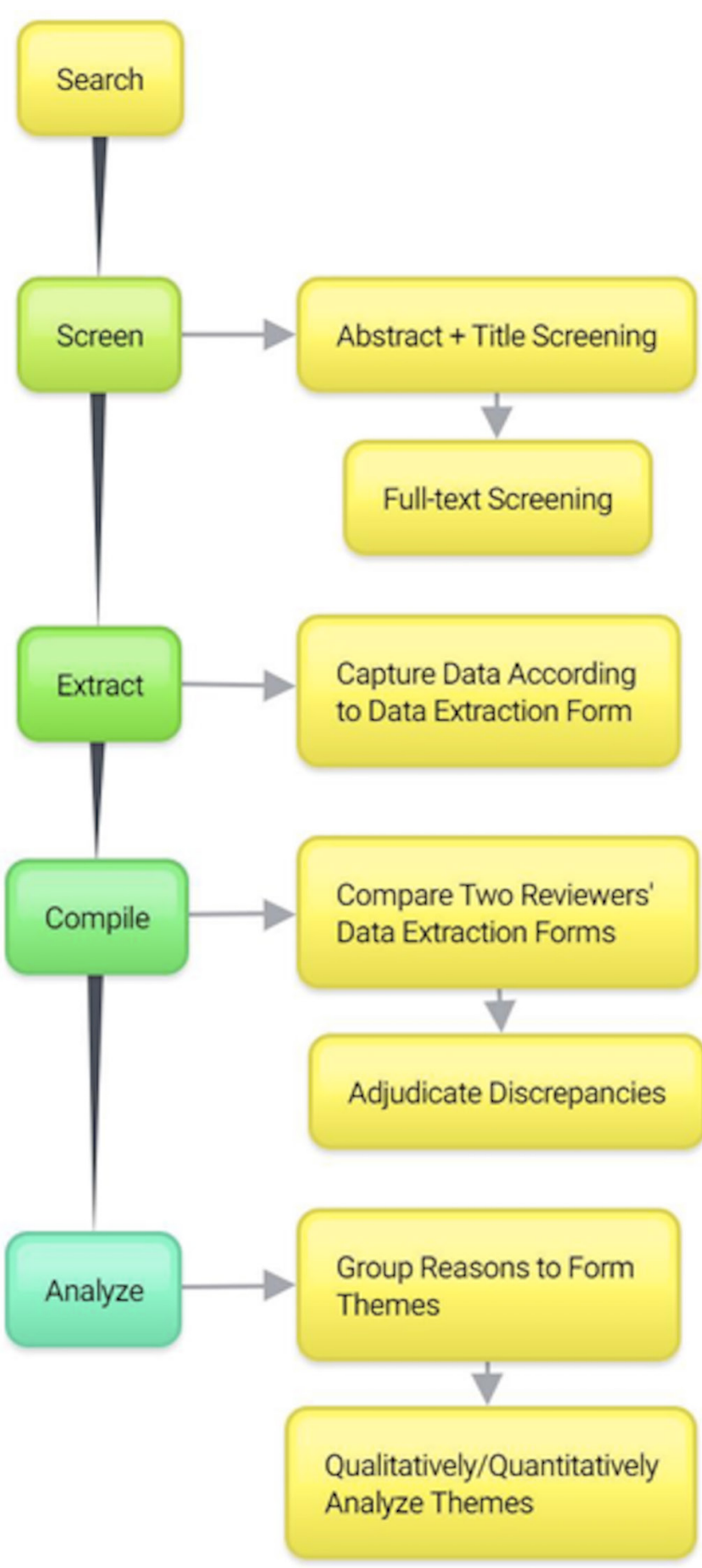

Figure 1 Workflow.

the term 'men' will be excluded as will be specific issues that are outside the scope of this study or that are used to describe conditions that appear only in women. The search will exclude terms associated with specific gynaecological complaints, cosmetic procedure, domestic violence studies or psychosocial interventions. For a complete list of search terms, please see online supplemental appendix 1 .

The inclusion criteria for screening include interventional or observational trials, philosophical scholarship, reviews, editorials and commentaries that either perform 
or discuss women-only trials, published during or after 2000, and written in English. Exclusion criteria include other types of published papers, publications that are not full papers (including abstracts and conference posters), non-peer-reviewed literature, publications published prior to the year 2000 and publications authored in any non- English language. Further, literature pertaining to conditions or interventions that appear solely in women will be excluded, as will psychosocial, physiotherapy or cosmetic interventions.

The data extraction process will reflect our three questions. First, a simple count of the number of women-only RCTs that have been conducted in conditions affecting men and women. Second, within these articles, how many offer an explicit reason for conducting a women-only trial. Third, an analysis of those reasons using a grounded theory approach to organised specific reason statements into themes.

Our methodology regarding identification and analysis of reasons has been adapted from a methodology developed by Strech and Sofaer for the systematic review of bioethical literature. ${ }^{8}$ This method is intended to address broad and complex literature by focusing solely on the reasons given for particular decisions on stances. As described in Strech's methodology, a reason can be defined as: an explanation given for the views that a position, action or policy is or is not permissible or warranted. ${ }^{9}$ In the context of this study, a reason is a segment of text that provides at least one explicit coherent justification for either the proposition that performing a women-only RCT is warranted or alternatively is not warranted. For example, 'a women-only RCT would be justified because women were underrepresented in earlier trials' would be an archetypal reason. As per scoping review methodology, there will not be an analysis of the quality of the papers in which reasons appear, and the strengths of the reasons will not be evaluated. This decision is made in an attempt to limit bias during the assessment process.

The analytical methods of this study incorporate a mixed methods review, ${ }^{10}$ in which a systematic and quantitative process allows for the comprehensive identification of relevant passages of text, which are then analysed qualitatively. Reasons will be extracted from the text and coded into themes by two reviewers. Interrater reliability will be captured and reported.

It is expected that there will be heterogeneity in the reasons given for why a women-only RCT may be justified. Such reasons may include, but they are not limited to:

- Conditions that have been well studied in men but not in women.

- Trials that enrolled significantly fewer women than men.

- Trials that were performed on women in the past of poor quality (confounding variables, not generalisable, inadequate power, falsification of results etc).

- Conditions where men respond favourably to treatment options and women do not.
- Conditions where there are sex differences in prevalence, diagnosis, severity or outcomes.

- Conditions that disproportionately affect women.

These concepts will not be defined at the data extraction phase, as there may be multiple versions of the same concept. Instead, through using the framework method, ${ }^{11}$ it is expected that themes will emerge during the data synthesis stage.

Data extraction is expected to be complete by September 2022. Data synthesis is expected to be complete by September 2023.

\section{Patient and public involvement}

No patient or public involvement is required in the design of this study, as the primary research question under investigation-how women-only RCTs are justified-is retrospective in nature and occurs before and without patient participation or experience.

\section{ELIGIBILITY CRITERIA}

\section{Study characteristics}

This scoping review will include RCTs pertaining to the reasons given to justify randomisation in women-only clinical trials. Only RCTs including adult women as participants will be included.

\section{Setting and time frame}

This review will include papers produced in 2000 or later to limit the search to the most modern context. The dates of the papers selected for inclusion will be captured during data extraction. Inpatient or outpatient RCT settings will be included.

\section{Report characteristics}

Articles written in English will be included, reflecting the authors' language of facility. Only completed articles that have been published will be included. Abstracts and articles in press will be excluded.

\section{Information sources}

Electronic databases, grey literature, bibliographies and authors of selected papers will be searched. Electronic databases of the medical literature that will be searched include MEDLINE, EMBASE, Cochrane Database of Systematic Reviews, Cochrane Clinical Trials Register, Web of Science Proceedings, ClinicalTrials.gov; databases of philosophical literature including Philosopher's Index, Phil Papers, JSTOR, Periodicals Archive Online, Project MUSE and the National Reference Centre for Bioethics. The University of Ottawa library catalogue will be searched, and suggestions will be solicited from experts in the fields of medical ethics and other philosophy and clinical trial methodologies. A number of related search terms were combined to create the original set of search criteria. Please see online supplemental appendix 1 for the full search terms. The search terms will be reviewed after identifying fundamental papers to ensure that the search methodology is appropriately inclusive. 


\section{Box 1 Data extraction form}

Article number
Last name of first author
Title
Journal
Year
Regarding the trial: PICO
Patients:
Intervention:
Comparator:
Outcome:
Is there a reason or reason(s) given to justify performance of a woman-
only randomised clinical trial? Yes or no
Extracted text(s) offering the reason:

\section{DATA ITEMS}

The following bibliographic information from the included studies will be collected: the first author, title of the paper, the journal in which it was published and the year of publication. In-text citations of reasons given to justify a women-only RCT will be collected based on the following formulation: 'a women-only RCT is justified if $\mathrm{X}$ ' or 'if X does not apply, then a women-only RCT is unethical' or a variation of this structure. This pattern is followed as there are nearly infinite ways to state a reason.

\section{DATA COLLECTION AND ANALYSIS}

Identified trials will be imported into the Covidence systematic review management software and screen for selection by two reviewers using titles and abstracts; this process will be documented in a flow diagram according to the Preferred Reporting Items for Systematic Reviews and Meta-Analyses for Scoping Reviews criteria. Title and abstracts will be screened according to inclusion/ exclusion criteria by two reviewers, with discrepancies adjudicated by consensus. Next, full-text screening will occur by two reviewers, with discrepancies adjudicated by consensus. Data will be extracted independently by two reviewers using the standardised form described above (box 1). Data extraction will be tested with 10 studies, and the results of these extractions will be compared for homogeneity. New instructions will then be given to increase interrater reliability. On attaining high levels of agreement, full data extraction of all included trials will begin. We will identify the conditions in which womenonly trials are being proposed or conducted, whether a specific rationale or reason is offered for this design and what that reason/rationale might be. The set of reasons will then be mapped onto a set of themes. At the title/ abstract screening and full-text screening phases, two independent assessors will determine whether a study meets inclusion criteria. In the event of a conflict, the conflict will be resolved via discussion or appeal to a third reviewer in the case of persistent disagreement. All phases of data collection will be completed by AM, MS and BD.

\section{DATA SYNTHESIS}

Reviewers will meet after data extraction is complete to combine the extracted reasons using a framework approach, based on methods from Gale et al. ${ }^{11}$ Potential reasons given earlier are subjected to modification as data are being synthesised. Reasons will be grouped by themes. A quantitative review will be produced that identifies if these studies are being conducted, if reasons are being offered to justify their conduct and what those reasons may be. A narrative review will be produced as well. The identification of themes will lead to reconsideration of the relevant literature, further refinement of the themes and the generation of conclusions.

As outlined in the methodology developed by Strech and Sofaer ${ }^{9}$ for a systematic review of bioethical literature, there will not be a risk of bias assessment.

\section{Presentation of results}

We will present a list of themes, reflecting groups of reasons, analysed by the frequency of their use, use over time and associations with author type. From these themes, conclusions will be drawn about the scope of the field regarding the ethical justification of women-only RCTs. These conclusions will then be used to inform future work on developing guidelines and translating them into practice.

\section{ETHICS AND DISSEMINATION}

Research ethics board approval is not required for this study as there is no participant involvement. We commit to publishing this protocol as well as the results from this study.

This study is part of a larger project looking at whether landmark carotid endarterectomy trials to treat carotid stenosis should be replicated in women-only. This project aims to inform a discussion surrounding whether a women-only RCT of carotid intervention for women with symptomatic high-grade carotid stenosis would be ethically justified.

Women's participation in medical research has a complicated history $^{12-14}$ that should serve as a reminder to ensure the same mistakes are not made again. To help prevent women being subject of unnecessary or potentially harmful RCTs are a result of either historical underrepresentation or unnecessary reproduction of trials, it is important to capture the reasons given for women-only RCTs. If a uniform set of reasons can be identified that appropriately justify ethical and scientific rationale for a women-only approach to RCTs, then these reasons may be used to create a set of criteria that can be applied to every women-only RCT proposed in future.

Contributors MS is the guarantor of the review. AM, BD and MS contributed to the design of the study and writing of the protocol. AM, BD, MS, OB, AD, MF, VY, DF, SG, $\mathrm{DD}$ and RF assisted in editing of the protocol. All authors gave final approval for the work.

Funding This work was supported by a Seed Catalyst Grant from the Heart and Stroke Foundation of Canada (2018) grant number 000 and by a Pilot Grant from the Department of Medicine, University of Ottawa (2019) grant number 000. 
Competing interests None declared.

Patient consent for publication Not required.

Provenance and peer review Not commissioned; externally peer reviewed.

Supplemental material This content has been supplied by the author(s). It has not been vetted by BMJ Publishing Group Limited (BMJ) and may not have been peer-reviewed. Any opinions or recommendations discussed are solely those of the author(s) and are not endorsed by BMJ. BMJ disclaims all liability and responsibility arising from any reliance placed on the content. Where the content includes any translated material, BMJ does not warrant the accuracy and reliability of the translations (including but not limited to local regulations, clinical guidelines, terminology, drug names and drug dosages), and is not responsible for any error and/or omissions arising from translation and adaptation or otherwise.

Open access This is an open access article distributed in accordance with the Creative Commons Attribution Non Commercial (CC BY-NC 4.0) license, which permits others to distribute, remix, adapt, build upon this work non-commercially, and license their derivative works on different terms, provided the original work is properly cited, appropriate credit is given, any changes made indicated, and the use is non-commercial. See: http://creativecommons.org/licenses/by-nc/4.0/.

\section{ORCID iDs}

Ainsley Matthewson http://orcid.org/0000-0002-6577-3042

Vignan Yogendrakumar http://orcid.org/0000-0001-8814-6853

Dean A Fergusson http://orcid.org/0000-0002-3389-2485

\section{REFERENCES}

1 Kabisch M, Ruckes C, Seibert-Grafe M, et al. Randomized controlled trials: part 17 of a series on evaluation of scientific publications. Dtsch Arztebl Int 2011;108:663-8.
2 Melloni C, Berger JS, Wang TY, et al. Representation of women in randomized clinical trials of cardiovascular disease prevention. Circ Cardiovasc Qual Outcomes 2010;3:135-42.

3 Marulanda-Londoño E, Chaturvedi S. Carotid stenosis in women: time for a reappraisal. Stroke Vasc Neurol 2016;1:192-6.

4 Bereznyakova O, Dewar B, Dowlatshahi D, et al. Benefit of carotid revascularisation for women with symptomatic carotid stenosis: protocol for a systematic review. BMJ Open 2019;9:e032140.

5 Liu KA, Mager NAD, DiPietro Mager NA. Women's involvement in clinical trials: historical perspective and future implications. Pharm Pract 2016;14:708.

6 Lippman A. The inclusion of women in clinical trials: are we asking the right questions? [Internet]. Canada: Women and Health Protection, 2006: 38. https://whp-apsf.ca/pdf/clinicalTrialsEN.pdf

7 Peters MDJ, Godfrey CM, Khalil H, et al. Guidance for conducting systematic scoping reviews. Int J Evid Based Healthc 2015;13:141-6.

8 Sofaer N, Strech D. The need for systematic reviews of reasons. Bioethics 2012;26:315-28.

9 Strech D, Sofaer N. How to write a systematic review of reasons. J Med Ethics 2012;38:121-6.

10 Grant MJ, Booth A. A typology of reviews: an analysis of 14 review types and associated methodologies. Health Info Libr J 2009:26:91-108.

11 Gale NK, Heath G, Cameron E, et al. Using the framework method for the analysis of qualitative data in multi-disciplinary health research. BMC Med Res Methodol 2013;13:117.

12 Vargesson N. Thalidomide-induced teratogenesis: history and mechanisms. Birth Defects Res C Embryo Today 2015;105:140-56.

13 Goldzieher JW, Moses LE, Averkin E, et al. A placebo-controlled double-blind crossover investigation of the side effects attributed to oral contraceptives. Fertil Steril 1971;22:609-23.

14 Vargas T. Guinea pigs or pioneers? How Puerto Rican women were used to test birth control pill, 2020. Available: https://www. washingtonpost.com/news/retropolis/wp/2017/05/09/guinea-pigsor-pioneers-how-puerto-rican-women-were-used-to-test-the-birthcontrol-pill/ 